\title{
Cardiopulmonary arrest in general wards: a retrospective study of referral patterns to an intensive care facility and their influence on outcome
}

\author{
S Premachandran, A D Redmond, R Liddle, J Mary Jones
}

Accident and

Emergency

Department, St

George's Hospital NHS

Trust, Blackshaw

Road, London SW17

S Premachandran

North Staffordshire

Hospital NHS Trust,

Stoke-on-Trent

A D Redmond

Resuscitation

Training,

Wythenshawe Hospital,

Manchester

R Liddle

Mathematics

Department, Keele

University

$\mathrm{J}$ Mary Jones

Correspondence to:

Mr S Premachandran, senior

registrar, Accident and

Emergency Department, St

George's Hospital NHS

Trust, Blackshaw Road,

London SW17 ORE

Accepted for publication

7 August 1996

\begin{abstract}
Objective-To analyse the effect on outcome of referral to specialist facilities after cardiopulmonary arrest in a general ward.

Methods-A retrospective analysis of resuscitation records of 743 patients in whom cardiopulmonary resuscitation was performed in a general ward between 1988 and 1992. After successful initial cardiopulmonary resuscitation, patients were identified as transferred to coronary care unit (CCU) or intensive care unit (ITU), or as staying in a general ward.
\end{abstract}

Main outcome measure-Survival to discharge home.

Results-There were 322 initial survivors, of whom 148 ( $20 \%$ of the overall total) survived to be discharged from hospital; $63 \%$ of those transferred to CCU and $48 \%$ of those transferred to ITU survived to discharge, compared with $28 \%$ of those who stayed on the ward $(P<0.001)$. Of those aged less than 65 years, $75 \%$ survived to discharge after transfer to $\mathrm{CCU}$ and $54 \%$ after transfer to ITU, compared with $44 \%$ of those who stayed on the ward $(P=0.023)$; the respective figures for those over 65 years were: CCU $25 \%$, ITU $34 \%$, ward $25 \%(P=0.014)$. Only half of those aged more than 65 years were transferred to a specialist facility, compared with $90 \%$ of those aged less than 65.

Conclusions-Transfer to a specialist care facility after resuscitation from cardiopulmonary arrest has an influence on outcome. Age as an independent factor is not an appropriate criterion to use in deciding on transfer. The decision to arrange transfer must always be taken by the most experienced person available, and in line with peer reviewed guidelines. (F Accid Emerg Med 1997;14:26-29)

Keywords: cardiopulmonary arrest; transfer to specialist facility; outcome

The return of spontaneous circulation does not necessarily mean the end of a successful resuscitation after cardiopulmonary arrest: more likely, it marks the start of prolonged and difficult postresuscitation care. ${ }^{1}$ The outcome of cardiopulmonary resuscitation in general wards is poor, ${ }^{2}$ and the European Resuscitation Council recommends that all patients resusci- tated from cardiopulmonary arrest should be admitted to an intensive care facility. ${ }^{3}$

Despite the large number of hospital patients on whom cardiopulmonary resuscitation is carried out each year, there have been few studies of postresuscitation care and its influence on outcome. ${ }^{4}$

We investigated the influence of referral pattern to an intensive care facility on outcome from cardiopulmonary arrest.

\section{Methods}

We carried out a retrospective study of patients resuscitated from cardiopulmonary arrest in general wards in a large district general hospital with a regional cardiothoracic centre.

We examined the resuscitation record forms for the years 1988 to 1992 . Cardiopulmonary arrest was defined as the sudden cessation of circulation or respiration, requiring initiation of cardiopulmonary resuscitation. ${ }^{5}$ For the purpose of this study we included only those cases of cardiopulmonary arrest that occurred in general wards. Cardiopulmonary arrest treated initially in the accident and emergency department, intensive care unit (ICU), or coronary care unit (CCU), and cardiopulmonary arrest in children were excluded from the study. Our aim was to analyse the referral patterns from "non-specialist" facilities to an intensive care environment.

It has been established practice at the study hospital for over 20 years for the senior doctor present at a cardiopulmonary arrest to complete a summary sheet immediately afterwards.

All completed forms were collated by the hospital resuscitation committee. When the seal on the resuscitation box was broken it had to be sent to the pharmacy for restocking and a new form supplied. This ensured a complete set of records.

This provided information on the place of arrest, type of arrest, immediate outcome, and whether the patient stayed in the ward or was transferred to ICU or CCU for further care. Additional information on those who survived to discharge was obtained from hospital records and the hospital computer PAS system.

Sixty five years of age is widely used as a cut off point for critical care facilities after cardiopulmonary arrest. We therefore looked specifically at the influence of patient age on outcome following cardiopulmonary arrest ${ }^{6}$ in 


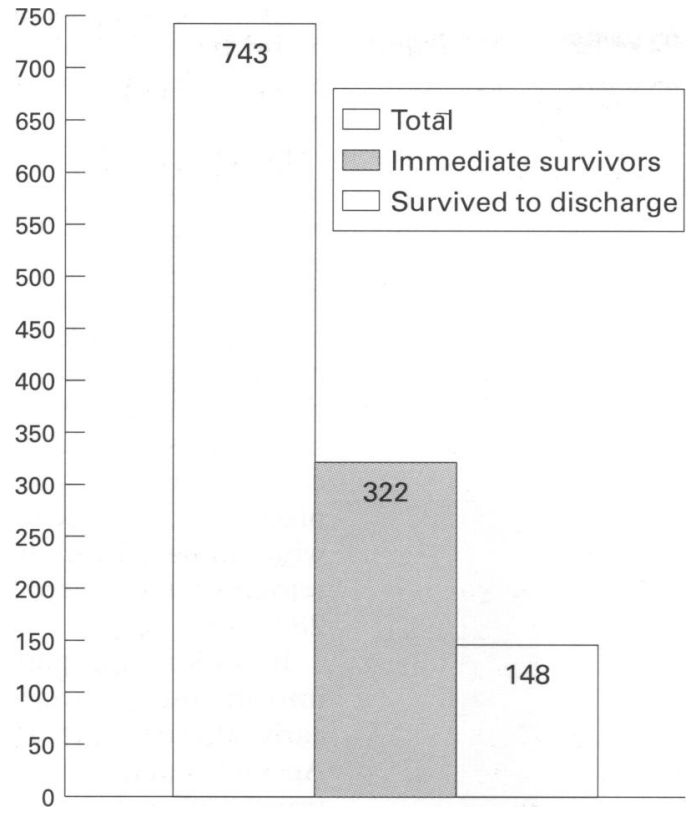

Figure 1 Survival after cardiopulmonary resuscitation.

those who stayed in the ward, or were transferred to CCU or ICU. Survival rates were compared using the $\chi^{2}$ test.

\section{Results}

In the 5 year period (1988 to 1992), 743 patients underwent cardiopulmonary resuscitation in a general ward; 322 (43\%) were initially successfully resuscitated. Of the 322 initial survivors, 148 (20\% of the total group) survived to be discharged from hospital (fig 1).

Survival outcome differed significantly $(P<$ 0.001 ) between CCU, ICU, and the ward: $63 \%$ of those transferred to CCU survived to discharge from hospital compared with $48 \%$ of those transferred to ICU and $28 \%$ of those who stayed in the ward (fig 2).

Of the 322 immediate survivors, $153(48 \%)$ were less than 65 years old, and 169 (52\%) more than 65 years old. Considering both age groups separately, the survival outcome differed significantly between CCU, ICU, and the ward. Among those less than 65 years, $75 \%$ of those transferred to CCU survived to discharge home compared with $54 \%$ of those transferred to ICU and $44 \%$ of those who stayed in the ward ( $P=0.023$ ) (figs 3 and 4$)$.

The survival rates were lower among those aged more than 65 years. However, half the patients transferred to CCU survived to be discharged home compared with $34 \%$ of those transferred to ICU and $25 \%$ of those who stayed in the ward $(P=0.014)$. Only half of those aged more than 65 years were transferred out of the ward to a specialist facility, in contrast to $90 \%$ of those aged less than 65 years of age, even though $52 \%$ of immediate survivors were more than 65 years of age (figs 5 and 6).

\section{Discussion}

In this retrospective study in a large district general hospital, outcome from cardiopulmonary arrest was related to postresuscitation
Cardiopulmonary arrest general wards

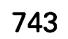

Immediate survivors

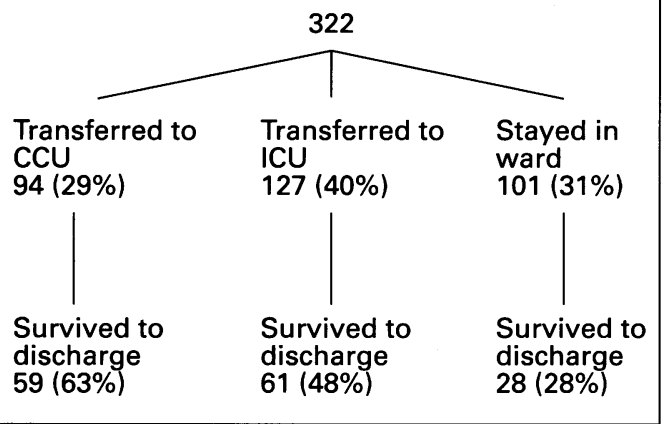

Figure 2 Referral pattern and survival after cardiopulmonary arrest in general ward.

Immediate survivors less than 65 years

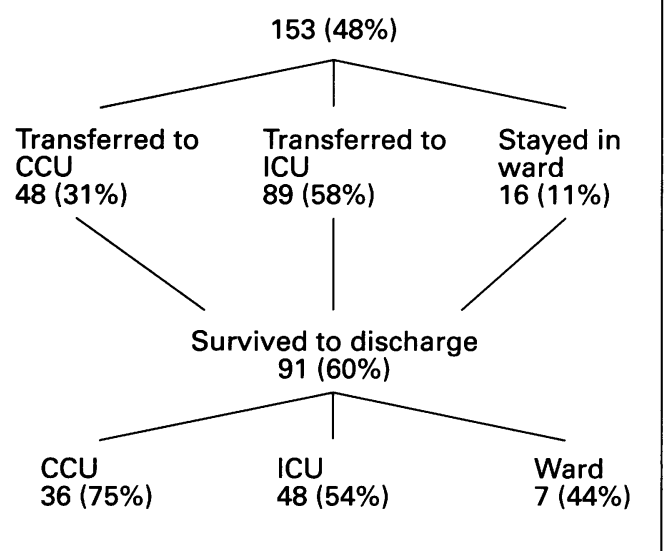

Figure 3 Referral pattern and survival in patients less than 65 years of age.

Immediate survivors more than 65 years

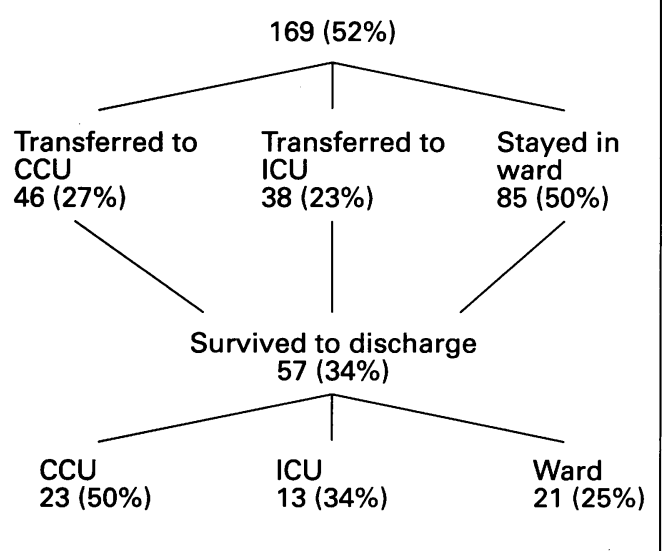

Figure 4 Referral pattern and survival in patients more than 65 years of age.

care. The overall survival rate in our study compared favourably with the findings of other studies. $^{6}$

However, survival rates differed significantly between those who were transferred to CCU 

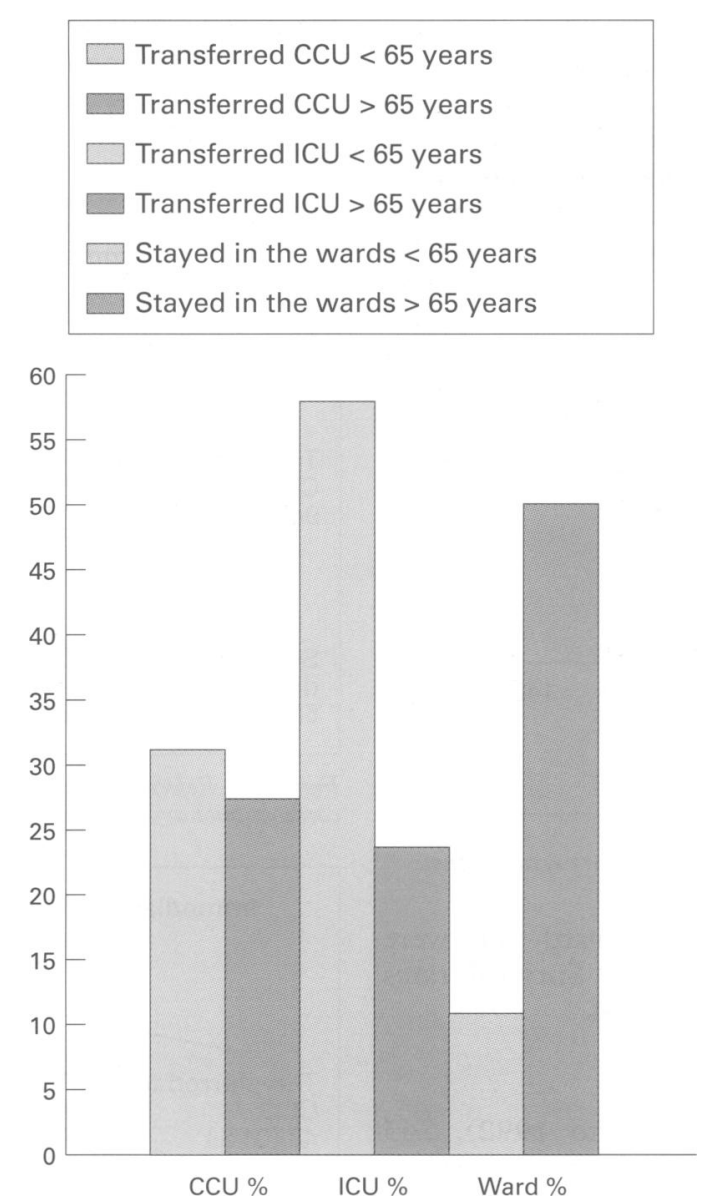

Figure 5 Comparison of referral patterns after cardiopulmonary arrest in a general ward in relation to age (<65 years; $>65$ years).

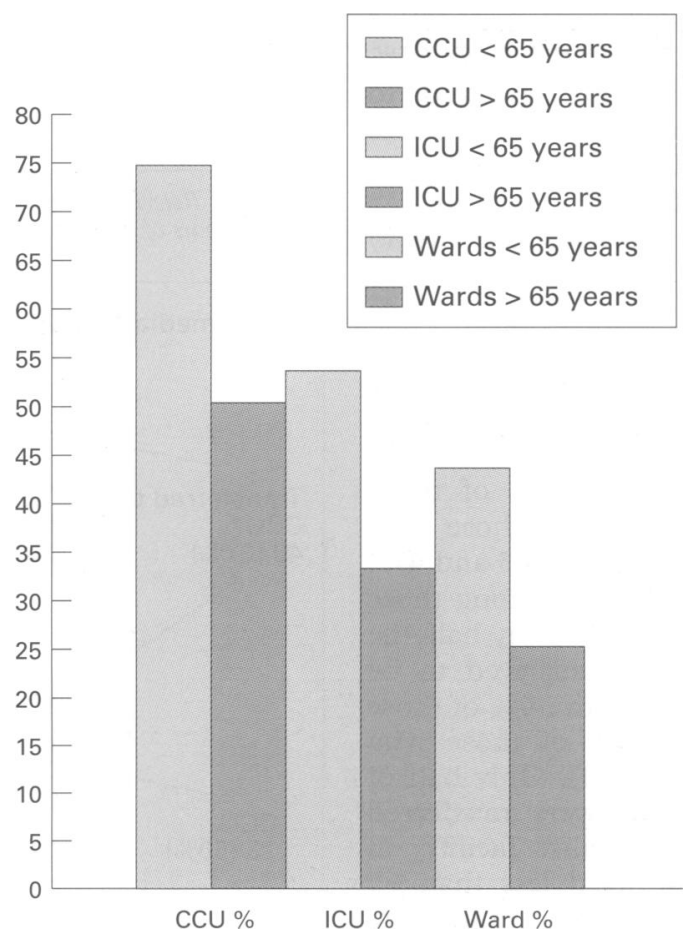

Figure 6 Comparison of survival to discharge after cardiopulmonary arrest in a general ward in relation to age ( $<65$ years; $>65$ years).

and ICU and those who stayed on the ward. Those who were transferred to CCU had the best chance of survival at all ages. This may represent patient selection as well as differ- ences in clinical management (for example, better pain control, early detection and treatment of dysrhythmias). ${ }^{6-8}$ This will be examined further.

Forty per cent of those who were resuscitated could not breathe spontaneously and were transferred to ICU for mechanical ventilation. Their survival rate was $48 \%$. One third of those over 65 years age who required mechanical ventilation survived to discharge home. There were no cases where patients were electively ventilated after cardiopulmonary arrest. Treatment in an ICU after cardiopulmonary arrest does seem to have a place in postresuscitation care, even for those who initially cannot breathe. Those who remained in the ward had a survival rate of only $28 \%$.

It is clear that patients were being selected into and out of specialist treatment areas in the early aftermath of a cardiopulmonary arrest. Age of the patients appeared to have influenced transfer of patients to an intensive care facility, and referral to a specialist facility did make a difference. A cut off point of 65 years would seem inappropriate for some patients, and the value of age alone as a criterion is doubtful. ${ }^{5}$

All patients who were resuscitated from cardiopulmonary arrest should be considered for admission to an intensive care or coronary care unit, ${ }^{4}$ as the survival rate for those who remain in the general wards is significantly lower at all ages. A decision to keep the patient in a ward should be reviewed in the same way as a DNR (do not resuscitate) order and must therefore be taken at consultant level after discussion with the relatives or the patient, or both. ${ }^{39}$

Predicting who will benefit most from specialist and intensive care after cardiac arrest is difficult. The absence of a measurable blood pressure when measured with a cuff is very unreliable and will not identify accurately those with a poor prognosis. ${ }^{10}$ Failure to breathe immediately, and even the age of the patient, cannot be relied upon to identify easily those most likely to benefit from intensive or other specialist facilities.

Anxieties about the use of intensive care facilities for postarrest patients may be unjustified, since half of the patients will live to be discharged home. The decision to use these expensive facilities can be difficult and often has to be balanced against the benefit to the patient.

There was obviously some selection of patients, but the point we wish to emphasise is that this was never laid down in any open policy. Junior doctors were making such decisions without reference to any agreed guidelines and on the spur of the moment. Possibly they did exclude patients with other serious medical conditions that would have prejudiced outcome on an intensive care facility, but this was never stated in the records. Detailed information about the medical status of the these groups would be too difficult to extract from the data available to us in this study. The results may not be explained entirely by selection, but given that failure to use such facilities has a significant effect on 
survival, it must be a decision that is always taken by the most experienced and in line with peer reviewed guidelines. We need to develop and evaluate criteria for admission to ICU and CCU after cardiopulmonary arrest. The decision to keep a patient on the ward will critically affect outcome. This is a decision that should be taken by a consultant. We recommend strongly that the use of intensive care facilities in the postarrest period should be monitored far more closely and recommendations developed. Our findings are to be followed up with a more detailed study to include information on the underlying disease and the nature of the cardiopulmonary arrest.

We are indebted to Wythenshawe Hospital resuscitation committee for their assistance and to the consultants for access committee for their assistance and to the consultants for access
to records of their patients; we thank Dr N H Brooks, consultant cardiologist, Dr K Shearer, hospital practitioner in cardiology, Wythenshawe Hospital, for their valuable comments.

1 Redmond AD. Postresuscitation care. ABC of resuscitation. London: BMJ Publishing Group, 1991:37-9.

2 Hershey CO, Fisher L. Why outcome of cardiopulmonary resuscitation in general wards is poor. Lancet 1982;i:31-4.

European Resuscitation Council Working Party. Adult ACLS: the European Resuscitation Council guidelines ACLS: the European Resuscit

4 Gustafson I, Edgren E. Brain-orientated intensive care after Gustafson I, Edgren E. Brain-orientated intensive care after
resuscitation from cardiac arrest. Resuscitation 1992;24:

5 Bedell SE, Delbanco TL. Survival after cardiopulmonary resuscitation in the hospital. N Engl J Med 1983;309:569608.

6 Tunstall-Pedoe H. The BRESUS study. BMJ 1992;304: 1347-51.

7 Levy RD, Rhoden WE. An audit of drug usage for in-hospital cardiopulmonary resuscitation. Eur Heart J 1992;13:1665-8.

8 Thomassen A, Wernberg M. Prevalence and prognostic significance of coma after cardiac arrest outside ICU \& CCU. Acta Anaesthesiol Scand 1979;23:143-8.

9 Doyal L, Wilsher D. Withholding cardiopulmonary resuscitation:proposals for formal guidlines. BMJ 1993; 306:1593-6.

10 Ryan BP, Redmond AD. When to stop resuscitation-the significance of cuff BP. Arch Emerg Med 1991;8:177-81.

\section{ADVANCED LIFE SUPPORT GROUP}

\section{Major Incident Medical Management and Support} Courses: to be held in various centres throughout the UK in 1997. This is a three day course in "life support style" designed to train health service personnel to provide an effective response at a major incident.

Fee $£ 300.00$

Advanced Paediatric Life Support Courses: to be held in various centres throughout the UK in 1997. This is a three day course designed to provide training which will enable doctors and nurses to deal efficiently with all paediatric emergencies. The course is modular and has sections on paediatric resuscitation, serious illness and serious injury.

Fee varies according to centre. Range $£ 300.00-£ 350.00$.

Inquiries for both courses to: Jenny Antrobus

Advanced Life Support Group

Second Floor, The Dock Office,

Trafford Rd., Salford Quays,

Manchester M5 2XB

Tel 01618771999

Fax 01618771666 